\title{
Prise en charge du transplanté rénal en réanimation
}

\section{Current Management of Critically-IIl Kidney Transplant Recipient}

\author{
V. Brunot $\cdot$ R. Larcher $\cdot$ M. Amalric $\cdot$ L. Platon $\cdot$ J.-J. Tudesq $\cdot$ N. Besnard $\cdot$ D. Daubin $\cdot$ P. Corne $\cdot$ B. Jung $\cdot$ \\ K. Klouche
}

Reçu le 30 juillet 2018 ; accepté le 21 novembre 2018

(C) SRLF et Lavoisier SAS 2018

Résumé La transplantation rénale est la thérapeutique de choix de l'insuffisance rénale chronique au stade ultime, son usage est de plus en plus large. Les progrès réalisés dans les traitements immunosuppresseurs ont permis une amélioration de la durée de vie du greffon, mais au prix d'une augmentation des complications cardiovasculaires et infectieuses. Environ $5 \%$ des transplantés rénaux présentent des complications sévères qui nécessitent une prise en charge intensive. Elles sont principalement de cause infectieuse et dominées par la défaillance respiratoire aiguë. L'insuffisance rénale aiguë est commune, elle affecte la fonction du greffon à court et long termes. La prise en charge en réanimation de ces complications doit prendre en compte le terrain particulier du transplanté rénal et les effets délétères de l'immunosuppression, condition nécessaire à une amélioration de la mortalité qui reste à plus de $30 \%$.

Mots clés Transplantation rénale $\cdot$ Réanimation . Immunosuppression - Sepsis · Insuffisance respiratoire aiguë · Insuffisance rénale aiguë · Prise en charge

\footnotetext{
Abstract Renal transplantation represents the best therapeutic option for end-stage renal failure and is widely performed. Recent advances in immunosuppressive drugs have improved the graft survival but has led to an increase in cardiovascular and infectious events. Severe complications that need intensive care have occurred in about $5 \%$ of renal transplant recipients. Reasons of admission are frequently acute respiratory failure and causes are mostly infectious. Acute

V. Brunot · R. Larcher · M. Amalric · L. Platon · J.-J. Tudesq · N. Besnard · D. Daubin · P. Corne · B. Jung · K. Klouche $(\bowtie)$ Département de médecine intensive réanimation,

CHU Lapeyronie, 371, avenue du Doyen-Gaston-Giraud, F-34295 Montpellier, France

e-mail : k-klouche@chu-montpellier.fr

R. Larcher $\cdot$ B. Jung $\cdot$ K. Klouche

PhyMedExp, Inserm, CNRS, Montpellier,

F-34295 Montpellier, France
}

kidney injury is common and may impact both short- and long-term graft functions. Current management should take into account the specificity of kidney transplant recipient and adverse effects of immunosuppression in order to improve the ICU mortality which remains above $30 \%$.

Keywords Kidney transplantation · Intensive care · Immunosuppression - Sepsis - Acute respiratory failure · Acute kidney injury $\cdot$ Management

\section{Introduction}

La transplantation rénale est actuellement le traitement de choix de l'insuffisance rénale chronique (IRC) au stade ultime. En comparaison avec le traitement de suppléance extrarénale, elle assure une morbidité et une mortalité moindres, une meilleure qualité de vie avec un coût moins élevé $[1,2]$. Depuis ces dernières années, le nombre de transplantations rénales est en nette augmentation, estimé à 571,5 par million d'habitants par l'agence de la biomédecine [3]. C'est en grande partie le résultat d'une augmentation de la prévalence de l'IRC terminale et de la multiplication des transplantations à critères élargis (receveurs hyperimmunisés, donneurs et receveurs âgés) et à partir de donneurs vivants [4].

Certes, les progrès réalisés dans la gestion des traitements immunosuppresseurs (IS) ont permis une amélioration de la survie des greffons rénaux, mais ils se sont soldés par une augmentation du risque infectieux et oncologique $[1,2]$. La morbimortalité des transplantés rénaux (TRx), d'origine infectieuse mais surtout cardiovasculaire, reste en effet supérieure à celle de la population générale [1]. La survenue de ces complications revêt parfois des formes sévères qui nécessitent une prise en charge en réanimation, dont l'issue peut être défavorable [5-10]. Plus de $5 \%$ des TRx sont admis en réanimation, et environ deux tiers seulement vont survivre à cet épisode [5-10]. L'optimisation de la prise en charge et l'amélioration du pronostic du transplanté rénal 
(TR) nécessitent une connaissance précise de son terrain et des effets délétères de l'immunosuppression (IS).

Dans cette revue, nous nous proposons de décrire les principales complications sévères du TR, de discuter de leur prise en charge en réanimation et de la conduite à tenir visà-vis de l'IS, et d'en préciser le pronostic.

\section{Les particularités du transplanté rénal}

Le TR est et reste un insuffisant rénal chronique avec un passé plus ou moins long de traitement par épuration extrarénale (EER) (seules 10 à $15 \%$ des premières transplantations sont préemptives) [3]. Il cumule les comorbidités communément retrouvées chez l'insuffisant rénal chronique diabète sucré, hypertension artérielle, insuffisance cardiaque et troubles lipidiques - et les complications propres à l'IRC : une athéromatose accélérée, des calcifications vasculaires, une hypertrophie ventriculaire gauche et un état d'immunodépression latent $[11,12]$. La durée d'EER préalable à la transplantation est corrélée à une surmortalité dans l'année qui suit [13].

Les suites de la transplantation rénale sont marquées par la nécessité d'une IS qui dure le temps de celle-ci. Elle vise surtout à bloquer l'activation et la prolifération lymphocytaire [14]. La forte IS introduite lors de la phase d'induction (suites immédiates de la greffe) est réduite lors de la phase d'entretien (à distance de la greffe). Les traitements inducteurs incluent les anticorps polyclonaux antilymphocytes $\mathrm{T}$ (Thymoglobuline ${ }^{\circledR}$, ATG, sérum antilymphocytaire) entraînant une lymphopénie profonde qui se corrige lentement (quelques mois) et les anticorps monoclonaux (Simulect ${ }^{\mathbb{R}}$ ) dirigés spécifiquement contre le récepteur de l'interleukine2 (IL-2), non lymphopéniants. Les traitements d'entretien sont les inhibiteurs de la calcineurine ou anticalcineurine (surtout tacrolimus et ciclosporine), les inhibiteurs du signal de prolifération ou inhibiteur de la mammalian target of rapamycine (mTOR) [sirolimus ou évérolimus] et les inhibiteurs de la prolifération ou antimétabolite (azathioprine, mycophénolate mofétil). Le traitement IS repose sur une combinaison de ces molécules dépendante de l'ancienneté de la greffe et du niveau de tolérance. La corticothérapie est souvent intégrée au traitement IS sachant que les protocoles récents favorisent une stratégie d'épargne, voire de sevrage précoce de celle-ci [14]. Les rejets aigus cellulaires sont traités par de fortes doses de corticoïdes et en cas de corticorésistance par une cure d'anticorps antithymocytes. Les rejets aigus humoraux sont traités par des corticoïdes à fortes doses associés à des échanges plasmatiques, des perfusions d'immunoglobulines et des anticorps anti-CD20 (rituximab). Des protocoles de désensibilisation (échanges plasmatiques avec colonnes spécifiques) sont destinés, en outre, aux patients hyperimmunisés et à ceux bénéficiant de greffe $\mathrm{ABO}$ incompatible. Le tableau 1 résume les mécanismes d'action et les effets secondaires des principaux traitements IS. Les protocoles d'IS actuels sont devenus assez efficaces pour minimiser le risque de rejet aigu, autoriser des transplantations à haut risque immunologique et permettre une meilleure survie des greffons, mais au prix de la survenue d'infections parfois sévères $[15,16]$. En outre, les IS sont susceptibles d'induire des interactions médicamenteuses. Les anticalcineurines et les inhibiteurs du signal de prolifération sont métabolisés par la voie du cytochrome P450, voie commune à de nombreuses molécules : les macrolides, les inhibiteurs calciques et certains antifongiques (itraconazole, voriconazole) augmentent les taux circulants des anticalcineurines tandis que les inducteurs enzymatiques accélèrent le métabolisme des IS entraînant leur inefficacité.

La mortalité cardiovasculaire a baissé chez le TR, mais les décès d'origine cardiaque demeurent fréquents [17]. Un événement cardiovasculaire survient chez environ $10 \%$ des TRx avec une incidence qui s'élève de 6,5 à plus de $30 \%$ en cas d'antécédents ischémiques coronariens [18]. Le risque cardiovasculaire perdure du fait du terrain sousjacent, mais il peut être aggravé par le traitement IS $[17,18]$.

\section{Le transplanté rénal en réanimation}

La proportion de $\mathrm{TRx}$ admise en réanimation est variable selon les séries, de 4 à plus de $50 \%$. L'incidence des complications sévères est cependant difficile à quantifier, car les critères d'admission en réanimation sont très disparates. Dans certaines unités, les patients sont systématiquement admis dans les suites immédiates de la greffe. L'analyse d'une des premières cohortes montrait que près de $40 \%$ des $178 \mathrm{TRx}$ étaient admis en réanimation, mais seuls $24,7 \%(44 / 178)$ l'étaient si l'on excluait la période postopératoire immédiate [7]. Dans notre expérience, 5,6\% des 1015 TRx entre 1997 et 2007 l'avaient été soit 16/1000 patients par année [5]. Une étude similaire, portant sur 1355 transplantations réalisées entre 1992 et 2012, retrouvait 4,1\% d'admissions en réanimation [8]. La majorité des études postérieures à 2000 rapportent un taux d'admission inférieur à $10 \%$ suggérant une baisse de fréquence des admissions probablement due à la modification des protocoles d'IS et des prophylaxies antiinfectieuses $[5,6,9]$.

L'admission survient tardivement, généralement plus de six mois après la greffe. Des délais médians d'un an et jusqu'à deux ans après la transplantation ont été observés $[5,7,9]$.

Les motifs d'admission sont dominés par les complications médicales : dans une cohorte récente de 413 TRx, plus des deux tiers $(68,3 \%)$ des admissions étaient liées à une cause médicale, un peu moins d'un tiers à une chirurgie programmée et seulement $2 \%$ à une chirurgie non programmée [8]. Les infections notamment pulmonaires et les accidents 


\begin{tabular}{|c|c|c|c|}
\hline Classe thérapeutique / traitement & Utilisation & Mécanisme d'action & Principaux effets secondaires \\
\hline $\begin{array}{l}\text { Sérum antilymphocytaire : } \\
\text { antithymocytes, thymoglobulines, } \\
\text { ATG }\end{array}$ & $\begin{array}{l}\text { Induction } \\
\text { Rejet cellulaire } \\
\text { corticorésistant }\end{array}$ & $\begin{array}{l}\text { Déplétion lymphocytaire } \mathrm{T}\left(\mathrm{CD}_{2}-\right. \\
\left.\mathrm{CD}_{4}\right)\end{array}$ & $\begin{array}{l}\text { Fièvre, leucopénie, thrombopénie, } \\
\text { choc anaphylactique, maladie } \\
\text { sérique }\end{array}$ \\
\hline Basiliximab & Induction & $\begin{array}{l}\text { Anticorps humanisés spécifiques : } \\
\text { antirécepteurs de l'IL-2 }\end{array}$ & Très rares \\
\hline Tacrolimus & $\begin{array}{l}\text { Entretien } \\
\text { Rejet cellulaire }\end{array}$ & $\begin{array}{l}\text { Inhibe la production d'IL-2, diminue } \\
\text { la prolifération et la différentiation } \\
\text { lymphocytaire } \mathrm{T}\end{array}$ & $\begin{array}{l}\text { Néphrotoxicité, neurotoxicité }++, \\
\text { tremblements, HTA, hyperkaliémie, } \\
\text { diarrhée, diabète, interactions } \\
\text { médicamenteuses }\end{array}$ \\
\hline Ciclosporine & Entretien & $\begin{array}{l}\text { Inhibe la production d'IL-2, diminue } \\
\text { la prolifération et la différentiation } \\
\text { lymphocytaire } T\end{array}$ & $\begin{array}{l}\text { Néphrotoxicité, neurotoxicité, HTA, } \\
\text { hyperkaliémie, hirsutisme, } \\
\text { interactions médicamenteuses, } \\
\text { hypertrophie gingivale }\end{array}$ \\
\hline Sirolimus, évérolimus & Entretien & Bloque le cycle cellulaire & $\begin{array}{l}\text { Hyperlipidémie, troubles } \\
\text { de la cicatrisation, diarrhée, } \\
\text { œdèmes, pneumopathie, arthralgie, } \\
\text { leucopénie, anémie, syndrome } \\
\text { inflammatoire biologique }\end{array}$ \\
\hline $\begin{array}{l}\text { Antimétabolites : } \\
\text { Azathioprine, mycophénolate } \\
\text { mofétil (MMF) }\end{array}$ & Entretien & $\begin{array}{l}\text { Inhibition de la synthèse de l'ADN/ } \\
\text { ARN }\end{array}$ & $\begin{array}{l}\text { Leucopénie, thrombopénie, anémie, } \\
\text { pancytopénie, } \\
\text { Azathioprine : aplasie si allopurinol, } \\
\text { hépatoxicité } \\
M M F: \text { douleurs abdominales, } \\
\text { diarrhée, pancréatite aiguë }\end{array}$ \\
\hline
\end{tabular}

cardiovasculaires sont les principales pathologies rencontrées en réanimation [10].

Les complications cardiovasculaires incluant les accidents ischémiques coronariens et la défaillance cardiaque aiguë restent communes chez le TR. Elles ne présentent pas de grande particularité et ne seront pas décrites avec plus de précision. Toutefois, leur prise en charge, tout étant conforme à celle de tout patient de réanimation, doit intégrer la singularité du TR (terrain, athéromatose accélérée et effet lipidogène du traitement IS).

Le tableau 2 résume l'ensemble des motifs d'admission et des diagnostics en réanimation.

\section{Complications infectieuses}

Plus de $80 \%$ des prises en charge en réanimation sont liées à une infection. Le TR est à la fois exposé à diverses sources infectieuses (communautaire et nosocomiale) et soumis à l'immunodépression (Fig. 1). L'immunodépression résulte, outre les facteurs peu spécifiques comme l'âge, la prédisposition génétique, le statut nutritionnel et les désordres métaboliques associés (diabète, cirrhose ou l'urémie), de l'IS induite par le traitement antirejet.
Le risque infectieux est en effet largement conditionné par la dose, la séquence et la durée de l'IS [16]. Le niveau de l'IS est dépendant du délai écoulé par rapport à la transplantation que l'on peut découper en trois phases (Fig. 2). La première phase correspond au premier mois postgreffe. C'est la période des infections nosocomiales : pneumopathies, infections urinaires sur sonde, infections liées aux cathéters, infections de paroi. Les infections transmises par le donneur ou par le liquide de conservation surviennent dans cette période (délai médian de 25 jours). La deuxième phase, du premier au sixième mois, est celle où l'IS est maximale favorisant la survenue des infections opportunistes et la réactivation d'infections latentes. La prophylaxie ordinairement maintenue jusqu'au sixième mois empêche le plus souvent le développement de pneumocystose et d'infections à cytomégalovirus (CMV). Toutefois, plusieurs infections opportunistes peuvent survenir notamment en cas de forte IS. Ce sont principalement des infections à mycobactéries (Mycobacterium tuberculosis) disséminées ou extrapulmonaires, à Nocardia (abcès cérébraux et atteinte pulmonaire), à cryptocoques (méningite, pneumopathie), à toxoplasme (atteinte cérébrale, mais également pulmonaire et myocardique) ainsi que des colites à Clostridium difficile. Au-delà du sixième 


\begin{tabular}{|c|c|c|}
\hline Motif d'admission & Étiologies les plus fréquentes & À évoquer de principe \\
\hline Détresse respiratoire aiguë & $\begin{array}{l}\text { 1- OAP cardiogénique ; cardiopathie ischémique, } \\
\text { hypervolémie secondaire à l'insuffisance rénale } \\
\text { 2- Pneumopathie infectieuse bactérienne ou virale }\end{array}$ & $\begin{array}{l}\text { - Pneumopathie médicamenteuse : sirolimus } \\
\text { - Pneumocystose pulmonaire si pas de prophylaxie }\end{array}$ \\
\hline État de choc & $\begin{array}{l}\text { Septique : } \\
\text { bactériémie, pneumopathie, infection urinaire, } \\
\text { infection liée aux cathéters... } \\
\text { Cardiogénique : } \\
\text { ischémie coronaire }\end{array}$ & - Pyélonéphrite aiguë du greffon \\
\hline Insuffisance rénale aiguë & $\begin{array}{l}\text { 1- IRA septique } \\
\text { 2- Hypovolémie/diarrhée } \\
\text { 3- Toxicité des ACN (anticalcineurines) }\end{array}$ & $\begin{array}{l}\text { - Thrombose artérielle ou veineuse du greffon } \\
\text { - Toxicité médicamenteuse } \\
\text { - Obstruction : sténose des voies urinaires, } \\
\text { caillotage, obstruction sonde JJ } \\
\text { - MAT (microangiopathie thrombotique) } \\
\text { - Récidive de la néphropathie initiale } \\
\text { - Néphropathie tubulo-interstitielle à virus BK }\end{array}$ \\
\hline Troubles de la conscience & $\begin{array}{l}\text { 1- Encéphalopathie métabolique et toxique } \\
\text { 2- AVC ischémique ou hémorragique } \\
\text { 3- Infection }\end{array}$ & $\begin{array}{l}\text { - Méningoencéphalite virale } \\
\text { - Méningite bactérienne, tuberculose, } \\
\text { Cryptococcus, Nocardia, Toxoplasma gondii } \\
\text { - Syndrome de l'encéphalopathie postérieure } \\
\text { réversible (PRES) }\end{array}$ \\
\hline
\end{tabular}

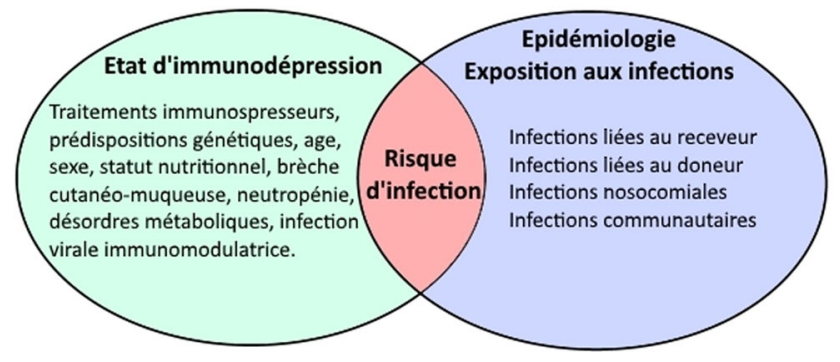

Fig. 1 Le transplanté rénal est soumis à une immunosuppression et exposé à des infections diverses

mois, l'IS est généralement réduite. En conséquence, le risque de contracter une infection opportuniste diminue laissant la place aux infections communautaires : pneumopathies bactériennes et pyélonéphrites du greffon. Des pneumocystoses pulmonaires et des infections à CMV peuvent cependant survenir tardivement après l'arrêt de la prophylaxie. Les transplantés dont le niveau d'IS reste élevé du fait de la répétition de rejets aigus ou d'un état d'hyperimmunisation sont toujours susceptibles de présenter des infections opportunistes à distance de la greffe : pneumocystose et plus rarement aspergillose [19].

Dans l'appréciation du risque et de la nature de l'infection, d'autres facteurs interviennent : les stratégies (type et timing) de prévention des infections opportunistes (vaccination anti- pneumocoque, prophylaxie anti-CMV ou anti-Pneumocyctis jirovecii) et le type d'IS. Un protocole d'IS fondé sur le sirolimus ou sur la ciclosporine aggrave significativement le risque de survenue de pneumopathie par rapport à celui fondé sur le tacrolimus [19]. Certaines études récentes suggèrent cependant que le traitement IS aurait un effet protecteur en cas de bactériémie $[20,21]$ tandis que les infections virales par CMV aggravent l'état d'immunodépression [22].

Les complications infectieuses sont par ordre de fréquence : les pyélonéphrites aiguës du greffon, les pneumonies et les bactériémies [9]. Les pneumopathies infectieuses représentent néanmoins la principale cause de formes sévères de sepsis et le plus gros contingent d'admissions en réanimation $[5,8]$.

Les signes cliniques et radiologiques de l'infection sont souvent trompeurs $[5,17,23,24]$. L'origine est bactérienne dans $60 \%$ des cas $[9,10,24]$. Les infections virales à CMV ont été pendant longtemps une cause majeure de morbimortalité chez les TRs avec une incidence de 50 à $80 \%$ [24]. La mise au point de traitements efficaces incluant la chimioprophylaxie et le traitement préemptif par valganciclovir (en cas de greffon CMV positif chez un receveur négatif) a diminué de façon conséquente leur incidence. Elles restent malgré tout fréquentes avec des localisations et une symptomatologie diverses : asymptomatique (virémie objectivée par une PCR sanguine positive), maladie à CMV avec fièvre, troubles digestifs... cytolyse hépatique, leucothrombopénie ; 


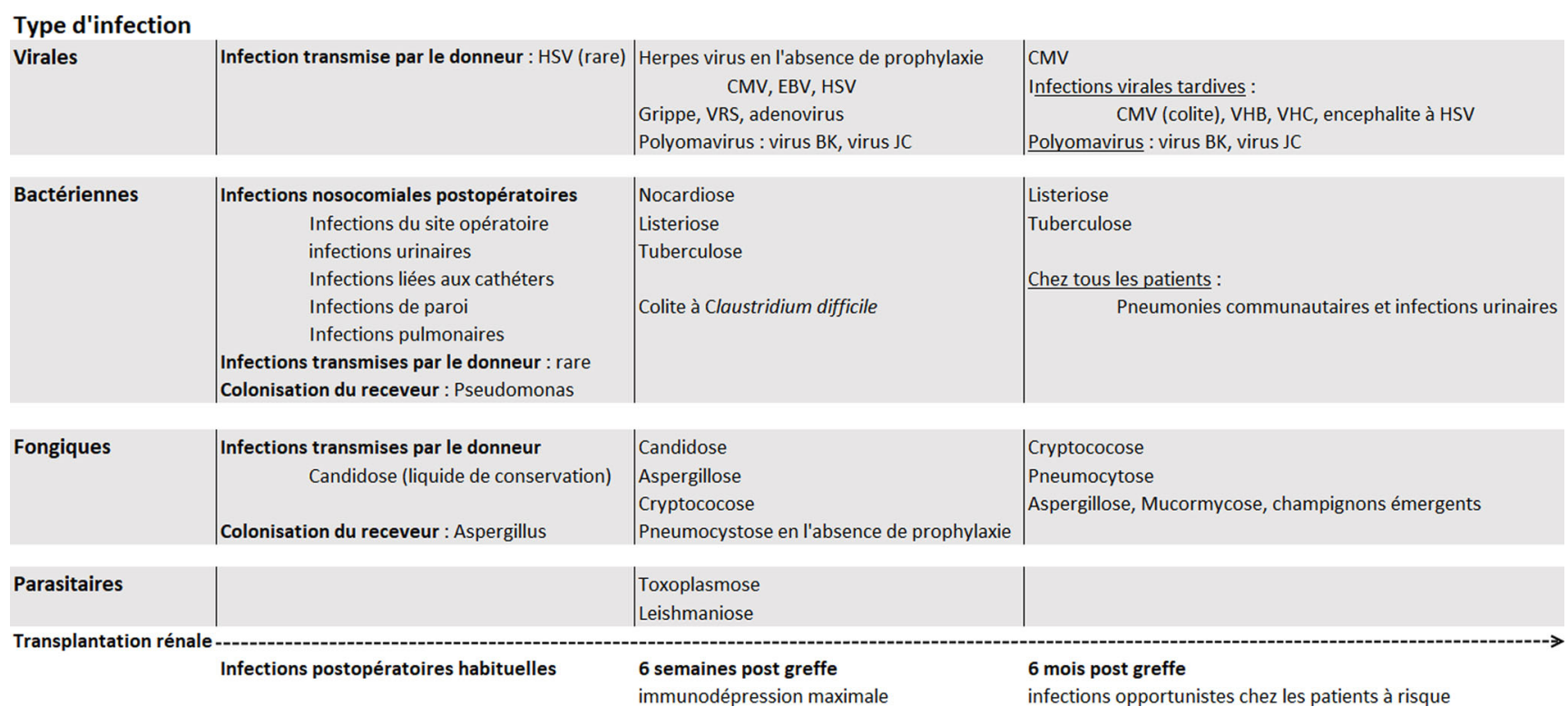

Fig. 2 Risque infectieux et principales infections en fonction du délai écoulé depuis la transplantation rénale (modifié d'après Fishman [16])

atteintes digestives (ulcérations et hémorragies), atteintes pulmonaires et co-infections. Dans une cohorte récente, l'infection à CMV atteignait $26,3 \%$ des TRs $(19,2 \%$ de maladie à $\mathrm{CMV}$ et $1 \%$ de pneumopathie) avec une mortalité de $0,3 \%$ [25]. La primo-infection à virus Epstein-Barr (EBV) est aussi à redouter surtout si le donneur est positif et le receveur négatif, responsable de tableau clinique sévère et de lymphome B, EBV induit, dont l'évolution explosive peut justifier une prise en charge en réanimation. Les cas d'hépatites fulminantes à HSV sont rares, mais de mauvais pronostic.

La prise en charge hémodynamique du choc septique ne présente pas de particularités. Le risque d'acidose métabolique hyperchlorémique secondaire à l'utilisation de volumes importants de sérum salé isotonique est prouvé dans ce contexte justifiant la recommandation de privilégier les solutés balancés comme produit de remplissage [26,27]. Les colloïdes de synthèse doivent être évités du fait d'un risque de toxicité avéré : néphrose osmotique et augmentation du recours à l'EER de la mortalité [28].

En cas d'infection sévère et/ou de choc septique, la conduite à tenir vis-à-vis de l'IS est délicate et loin d'être consensuelle. Elle doit tenir compte de la fonction de base du greffon, du risque immunologique du patient, de la cause et de la sévérité de l'infection. Il est bien entendu que la survie du patient prend le pas sur celle du greffon. Sans qu'il existe de preuves formelles, il semble légitime de diminuer l'IS chez les patients qui présentent une infection opportuniste prouvée. La baisse de l'IS est fortement recommandée et réalisée en cas d'infection à virus BK (PCR positive dans le sang ou les urines), mais elle est souvent laissée à l'appré- ciation du médecin en charge du patient en cas d'infections opportunistes telles qu'une réactivation virale à $\mathrm{CMV}$, à HSV, à EBV, ou une infection fongique. En cas d'infection grave communautaire ou nosocomiale, la gestion de l'IS est encore plus discutée et variable selon le patient et le centre. L'attitude générale est cependant celle de diminuer, voire d'arrêter l'IS pour renforcer les défenses immunitaires sachant que le sepsis induit une IS réduisant le risque de rejet aigu pendant l'épisode. L'étude rétrospective d'une cohorte de TRx avec une pneumonie bactérienne a montré que la diminution de l'IS n'était pas associée à une augmentation des épisodes de rejet aigu dans les deux ans suivant la réanimation [29]. Dans tous les cas, le maintien, l'allégement ou l'arrêt du traitement IS doivent être discutés au cas par cas en étroite collaboration avec les néphrologues responsables de la transplantation. Plusieurs stratégies sont proposées (Tableau 3). Une des plus usitées est celle de relayer l'IS par une corticothérapie systémique isolée. L'interruption du traitement IS à l'admission de $53 \mathrm{TRx}$ pour une détresse respiratoire aiguë et son relais par une corticothérapie à $1 \mathrm{mg} / \mathrm{kg}$ n'a entraîné aucun rejet au terme des six mois de suivi [30]. Le traitement IS peut être aussi modulé selon le monitorage du taux de lymphocytes T CD4 avec une interruption en cas de taux inférieur à 200 cellules $/ \mathrm{ml}$ et une réintroduction progressive à faibles doses dès que le taux est supérieur à 600 cellules/ml [31]. L'arrêt des inhibiteurs de la prolifération ou antimétabolites (azathioprine, mycophénolate mofétil) est réalisé par la majorité des équipes dans les infections sévères, surtout quand une leucopénie ou une lymphopénie s'y associent. 
Tableau 3 Une proposition de gestion des traitements immunosuppresseurs chez un transplanté rénal admis en réanimation pour sepsis ou choc septique

1- Contacter le néphrologue référent pour adapter

le traitement, évaluer le risque immunologique

2- Arrêt des antimétabolites, surtout en cas de leucopénie

3- Arrêt des inhibiteurs de la voie des mTOR surtout en cas de prise en charge chirurgicale ou de lésions cutanées, car ils retardent la cicatrisation. Arrêt si pneumopathie interstitielle

4- Maintien des anticalcineurines avec dosages sanguins

systématiques et réguliers. Arrêt si syndrome

de microangiopathie thrombotique

- Privilégier la voie orale

- Si la voie orale est impossible, ciclosporine i.v. en divisant la posologie par 3

- Adapter la posologie aux dosages plasmatiques per os (3 fois par semaine)

5- Maintien ou ajout des corticö̈des si arrêt

des antimétabolites (prednisolone $20 \mathrm{mg} / \mathrm{j}$ )

6- Faire un typage lymphocytaire (CD4/CD8) et un dosage pondéral des immunoglobulines pour apprécier le niveau d'immunodépression du patient

\section{Insuffisance respiratoire aiguë}

L'insuffisance respiratoire aiguë est la principale cause d'admission en réanimation ; plus de la moitié des admissions était liée à une détresse respiratoire aiguë dans l'étude multicentrique française [6]. L'origine de celle-ci est fréquemment non infectieuse durant la période postgreffe immédiate (œdème pulmonaire aigu cardiogénique) tandis qu'elle est majoritairement infectieuse à plus long terme. Environ $50 \%$ des infections sévères sont localisées au poumon $[5,6,9]$. Dans de rares cas, une origine toxique médicamenteuse est retrouvée.

La recherche d'une étiologie est capitale, car une recherche infructueuse est associée à une augmentation du recours à la ventilation mécanique invasive et à la mortalité [6,32]. L'appréciation de la fonction cardiaque par échocardiographie (ou un autre moyen d'exploration) doit être le préalable à cette enquête, car une défaillance cardiaque peut être méconnue et/ou pourrait être associée. Cette recherche, tout en tenant compte de la profondeur de l'immunodépression (cf. chapitre complications infectieuses), est complexe du fait de la variété et de la non-spécificité des tableaux clinico-radiologiques [24]. Une pneumonie bactérienne communautaire de début brutal est souvent secondaire à un pneumocoque $[6,9]$. Une toux persistante associée à un état clinique préservé malgré la profondeur de l'hypoxie et la sévérité de l'atteinte radiologique est très évocatrice d'une pneumocystose pulmonaire. Un début subaigu est plutôt en faveur d'une pneumopathie à $\mathrm{CMV}$, infection devenue rare avec la prophylaxie [24]. L'enquête étiologique doit être dans tous les cas systématique associant examen cytobactériologique des crachats, recherche d'antigènes urinaires (antigénuries pneumococoque et légionelle) et sanguins (antigénémie aspergillaire : galactomannane, PCR-CMV et autres virus), PCR Pneumocystis sur crachats induits et tomodensitométrie thoracique [33]. La fibroscopie bronchique avec lavage bronchoalvéolaire a un bon rendement diagnostique [39]. Dans une cohorte de 71 TRs, 63 des 91 (69\%) lavages réalisés ont permis de documenter l'infection : bactéries : 30 (33\%), Pneumocystis jiroveci : 21 ( $23 \%)$ et CMV : 25 (27\%) [10,34]. Cet examen permet de mettre en évidence des co-infections bactériennes notamment avec le CMV et le Pneumocystis et les atteintes toxiques avec l'analyse cellulaire et immunologique du liquide (Tableau 4). Il doit être largement réalisé chez le patient ventilé et chez le patient non ventilé avec les précautions d'usage. En effet, l'application d'un protocole standardisé

Tableau 4 Explorations du liquide bronchoalvéolaire en cas d'insuffisance respiratoire aiguë sévère

\begin{tabular}{|c|c|}
\hline Laboratoires & Examens \\
\hline \multirow[t]{7}{*}{ Bactériologie } & Examen direct \\
\hline & Coloration de Gram et culture \\
\hline & standard \\
\hline & Coloration par le May-Grünwald \\
\hline & Giemsa \\
\hline & Germes intracellulaires, PCR \\
\hline & Nocardia et Actinomyces \\
\hline \multirow[t]{8}{*}{ Virologie } & Virus respiratoires : PCR \\
\hline & Grippe A et B, parainfluenzae \\
\hline & de types 1 à 4, VRS, hMPV, \\
\hline & coronavirus, adénovirus, Bocavirus, \\
\hline & Rhinovirus, Entérovirus \\
\hline & Herpes virus : PCR : CMV, HSV, \\
\hline & VZV, HHV6 \\
\hline & PCR adénovirus \\
\hline \multirow[t]{4}{*}{ Mycologie } & Galactomanane (antigène \\
\hline & aspergillaire) \\
\hline & Pneumocystis : examen direct, \\
\hline & $\begin{array}{l}\text { coloration de Gomori-Grocott et PCR } \\
\text { ou IF }\end{array}$ \\
\hline Parasitologie & Toxoplasmose \\
\hline \multirow[t]{2}{*}{ Mycobactérie } & Recherche BAAR, coloration \\
\hline & de Ziehl puis culture \\
\hline Anatomopathologie & Recherche d'une hémorragie intra- \\
\hline & alvéolaire (Score de Gold), dosage \\
\hline & quantitatif des lymphocytes (T4, T8) \\
\hline & néoplasie \\
\hline
\end{tabular}


incluant le lavage broncholoalvéolaire chez 40 TRx a permis d'obtenir une mortalité de moins de $15 \%$ [35]. Dans tous les cas, la documentation microbiologique permet d'ajuster le traitement anti-infectieux et améliore le pronostic [9]. L'exclusion d'une cause infectieuse doit faire rechercher une origine toxique comme les inhibiteurs des mTOR [36].

L'insuffisance respiratoire est souvent sévère associée à une hypoxémie profonde [30,37]. Elle nécessite une ventilation mécanique chez 60 à $80 \%$ des patients [5]. La ventilation non invasive (VNI) et l'oxygénothérapie à haut débit (OHD) permettent parfois de surseoir, voire d'éviter la ventilation invasive [38]. Les résultats prometteurs de la VNI objectivant une baisse de la mortalité chez le patient immunodéprimé n'ont pas pu être confirmés [39,40]. Il a été montré récemment que l'OHD nasal améliore la survie des patients immunocompétents ayant une insuffisance respiratoire aiguë hypoxique non hypercapnique ; les données chez le patient immunodéprimé restent néanmoins moins tranchées [41-43]. La comparaison d'une oxygénation par VNI ou par $\mathrm{O}_{2}$ nasal chez 374 patients immunodéprimés (dont 14 transplantés d'organe solide) avec une insuffisance respiratoire aiguë hypoxémique n'a pas montré de différence de mortalité à 28 jours $(24,1$ vs $27,3 \%, p=0,47)$ ni du taux d'intubation (38,2 vs $4,8 \%, p=0,20)$ [40]. L'analyse post hoc a montré que l'OHD en comparaison à l' $\mathrm{O}_{2}$ standard (91 patients dans chaque groupe) ne permettait pas non plus de réduire le taux d'intubation et la mortalité [43]. Dans une analyse post hoc similaire où 30 patients étaient traités par $\mathrm{O}_{2}$ seul, 26 avec de l'OHD seul et 26 par VNI et OHD ; ceux traités par VNI avaient la mortalité et le taux d'intubation les plus élevés [42]. La VNI et l'âge étaient les deux facteurs associés à la mortalité et à l'intubation endotrachéale [42]. Une étude prospective multicentrique de 1611 patients immunodéprimés avec insuffisance respiratoire aiguë hypoxémique $(8,8 \%$ de TRx) a montré que l'OHD diminuait significativement le taux d'intubation en comparaison avec $\mathrm{l}^{\prime} \mathrm{O}_{2}$ standard et la VNI, mais sans effet sur la mortalité [32]. Une méta-analyse récente groupant sept études a conclu que l'usage de l'OHD réduit la mortalité à court terme et le taux d'intubation sans augmentation de la durée de séjour [44]. Si plusieurs éléments semblent suggérer que la VNI aggrave le taux d'intubation et la mortalité chez ces patients, la place de l'OHD n'est pas définie. Toutefois, l'utilisation de l'OHD n'était pas supérieure à celle de l'oxygène standard en termes de mortalité dans l'essai contrôlé randomisé HIGH incluant 776 patients immunodéprimés avec insuffisance respiratoire aiguë [45].

Le traitement anti-infectieux ne présente pas de particularités en s'assurant du risque d'interactions avec les IS et la toxicité rénale. La prophylaxie par cotrimoxazole est efficace et évite généralement la survenue de pneumocystose mis à part quelques cas groupés régulièrement rapportés dans la littérature [46]. Le bénéfice prouvé de la corticothé- rapie dans la pneumocystose chez le patient VIH reste débattu chez le TR.

Les formes sévères d'insuffisance respiratoire aiguë nécessitant une ventilation invasive doivent bénéficier d'une ventilation protectrice. Ces formes restent cependant peu fréquentes $[37,47]: 51$ cas/100 000 patients par an dans une étude analysant 42190 PTR de 1994-1998 [48]. Cette population avait toutefois un risque plus élevé de développer un SDRA comparativement à la population générale [48]. Dans la cohorte LUNG SAFE de 2813 patients avec SDRA, l'analyse des 584 patients immunodéprimés a montré que sa survenue était plus fréquente, qu'une cause infectieuse était majoritairement retrouvée et que la VNI était utilisée de façon plus fréquente que chez les immunocompétents [49].

L'insuffisance respiratoire aiguë entraîne une mortalité de $22,5 \%$ [6]. Le pronostic est lié à la sévérité et à l'origine de l'atteinte respiratoire [6,32]. Une origine bactérienne ou fongique (pneumocystose, aspergillose et candidose) entraîne une mortalité significativement plus élevée que les autres étiologies [6]. La pneumocystose est responsable d'une mortalité d'environ $30 \%$, supérieure à celle observée chez les patients infectés par le VIH [6].

\section{Insuffisance rénale aiguë}

L'insuffisance rénale aiguë (IRA) concerne 15 à $30 \%$ des TRx admis en réanimation [10]. Dans une étude récente, l'analyse de $358 \mathrm{TRx}$ admis en réanimation trouvait une IRA chez 293 d'entre eux avec la répartition suivante selon la classification KDIGO : stade $1: 31,6 \% ; 2: 8,7 \%$ et 3 : $41,6 \%[50]$.

En postopératoire immédiat, la nécrose tubulaire aiguë (retard de reprise de fonction) est l'atteinte la plus fréquente, mais il faut éliminer systématiquement une obstruction de la vessie ou des voies urinaires, redouter une thrombose de l'artère ou de la veine du greffon, envisager un lâchage des sutures des voies urinaires (vessie ou uretère). Le rejet humoral hyperaigu ne survient pratiquement plus grâce au cross match préopératoire, mais un rejet aigu reste toujours possible.

À distance de la transplantation rénale, l'IRA est volontiers multifactorielle, le plus souvent septique, associée à des troubles hémodynamiques et/ou à un ou plusieurs néphrotoxique(s) surtout les anticalcineurines [51]. Une IRA satellite d'un rejet aigu est rarement rencontrée en réanimation du fait de l'immunodépression induite par le sepsis. Une IRA associée à une microangiopathie thrombotique (MAT) est une forme singulière y associant généralement une anémie hémolytique mécanique, une thrombopénie périphérique avec fréquemment une HTA. Les signes systémiques de MAT sont cependant souvent absents justifiant la réalisation d'une biopsie du greffon pour affirmer le diagnostic [52]. La MAT survient chez 1,5 à 14,8\% des TRx, essentiellement 
durant la période postopératoire [52]. Il faut distinguer les MAT récurrentes, qui sont la récidive sur le greffon d'une MAT connue (essentiellement des SHU atypiques), et les MAT de novo - les plus fréquentes - une entité plus hétérogène dominée par les causes médicamenteuses (anticalcineurines, inhibiteur des mTOR) [52]. La survenue d'une infection par le virus BK induit dans environ $5 \%$ des cas une néphropathie tubulo-interstitielle spécifique entraînant une perte progressive de fonction du greffon [53]. Le diagnostic repose sur la mise en évidence de decoy cells à l'analyse cytologique des urines fraîches, du virus par PCR dans les urines $\left(>10^{7}\right.$ copies $\left./ \mathrm{ml}\right)$ et dans le sang $\left(>10^{4}\right.$ copies/ $\mathrm{ml})$. Le traitement consiste avant tout à diminuer l'intensité de l'IS et à y associer parfois des antiviraux - cidofovir (néphrotoxique), brincidofovir - et/ou des immunoglobulines intraveineuses. Elle est cependant rarement une cause d'admission en réanimation. Les autres causes sont plus rares comme les rejets, les cancers, les infections à CMV...

Le recours à l'EER obéit aux mêmes règles que pour toute IRA, mais il est bien plus fréquent dans cette population. Près de $50 \%$ des TRx sont épurés au cours de leur séjour en réanimation alors que seulement 5 à $10 \%$ le sont dans la population générale de réanimation. Une atteinte respiratoire aiguë associée aggraverait le risque de recours à l'EER [37].

La survenue d'une IRA affecte le court et le long terme de la fonction du greffon. Trois mois après la sortie de réanimation, à peine plus d'un tiers $(37,5 \%)$ des patients recouvrent la fonction rénale antérieure, $20 \%$ gardent une fonction altérée et $20 \%$ sont pris en charge en hémodialyse chronique [6]. La survie du greffon est dépendante de la sévérité de la dysfonction rénale à l'admission [6].

\section{Complications neurologiques aiguës}

Les troubles neurologiques affectent plus de $80 \%$ des TRx [54], mais les formes sévères sont plus rares. Ils peuvent être secondaires à une infection, à une lésion vasculaire, cancéreuse, à une cause métabolique (en incluant la dysfonction du greffon) ou toxique $[54,55]$.

L'accident vasculaire cérébral est commun chez le TR, facilité par l'HTA, le diabète sucré et l'athérosclérose accélérée liée à l'IRC [55]. La ciclosporine, les inhibiteurs des mTOR et à moindre degré le tacrolimus entraînent souvent une hypercholestérolémie et une atteinte vasculocérébrale accélérée [55].

L'atteinte infectieuse neuroméningée se révèle parfois par un syndrome méningé, un coma fébrile, des convulsions ou des signes focaux, mais le tableau clinique est souvent fruste. Il existe une corrélation entre le micro-organisme responsable de l'infection et le temps écoulé depuis la transplantation. Les infections bactériennes spécifiques sont celles dues à Listeria monocytogenes, à mycobactéries et à Nocardia. Les atteintes dues à Listeria monocytogenes sont des méningites aiguës, des méningoencéphalites avec rhombencéphalite, des myélites ou des signes neurologiques focaux (abcès). Les mycobactéries sont plus rarement impliquées : tuberculome, méningite et myélopathie. Le diagnostic est souvent difficile, nécessitant la répétition des ponctions lombaires. La Nocardia est elle aussi rare affectant moins de $5 \%$ des TRx [56], donnant des tableaux de méningite subaiguë et chronique et de cérébrite. Les infections fongiques sont principalement liées à Cryptococcus neoformans et à Aspergillus fumigatus. Le cryptocoque donne des méningites subaiguës et chroniques avec des images caractéristiques à l'IRM. L'Aspergillus est responsable d'infections focales cérébrales (abcès frontopariétaux), mais aussi des lésions ischémiques et hémorragiques en cas d'aspergillose invasive. Les infections virales sont dominées par les infections à herpes virus (HSV-1) et à virus varicellozonateux (VZV). Les encéphalites et les polyradiculonévrites secondaires au CMV sont rares. Les infections à EBV donnent des méningites aseptiques, des convulsions, des encéphalites et des lymphomes posttransplantation. Une infection parasitaire surtout la toxoplasmose survient chez moins de $1 \%$ des TRx : méningite et abcès [57]. Les troubles cognitifs peuvent être dus au virus JC (Leucoencéphalopathie multifocale progressive [LEMP]).

Les atteintes néoplasiques cérébrales surviennent trois à quatre fois plus souvent que dans la population générale [58]. La fréquence des lymphomes posttransplantation est estimée à moins de $1 \%$ : lymphomes $\mathrm{B}$ à grandes cellules, lymphome de Burkitt et lymphome plasmablastique souvent viro-induits (EBV) imposant la réduction de l'IS et l'utilisation d'anticorps anti-CD20 (rituximab) [58].

Les atteintes toxiques sont celles dues aux corticoïdes et aux anticalcineurines. Le syndrome d'encéphalopathie postérieure réversible, ou posterior reversible encephalopathy syndrome (PRES), est un syndrome clinicoradiologique réversible rare, caractérisé par la survenue de céphalées, de troubles visuels, de convulsions qui sont liés à un œdème cérébral vasogénique de localisation principalement occipitale avec une IRM caractéristique. Il est associé à de nombreux facteurs, mais de façon quasi constante à une HTA sévère. Il est observé chez 0,5 à $5 \%$ des TRx majoritairement traités par anticalcineurines (tacrolimus, ciclosporine), plus rarement sous inhibiteur des mTOR [55].

Les troubles métaboliques sont liés aux troubles hydroélectrolytiques, aux complications sévères du diabète sucré et celles liées à l'IRC.

\section{Pronostic}

La mortalité à court terme des $\mathrm{TRx}$ en réanimation est très variable, oscillant de 12,8 à 42,6\% selon les séries et la sévérité $[8,50]$. Dans notre cohorte, le taux de mortalité était de $40 \%$, significativement plus élevé que celui d'une population 
générale de même gravité [5]. Les facteurs associés à la mortalité sont les scores de gravité à l'admission, le recours à la ventilation mécanique et l'état de choc. Parmi les défaillances d'organe, la défaillance respiratoire nécessitant une ventilation invasive est le facteur de plus mauvais pronostic indépendamment associé à la mortalité, à moindre degré l'IRA justifiant d'une EER [7-9,29,47]. Le sepsis est la deuxième cause de décès du TR après la mortalité d'origine cardiovasculaire. Des données contradictoires ont rapporté une mortalité plus faible en cas de sepsis sévère ou de choc septique par rapport à la population générale probablement du fait d'un suivi plus rapproché (diagnostic et traitements plus précoces) et de l'effet anti-inflammatoire, potentiellement bénéfique, de l'IS $[16,20,21,59]$. Cependant, une IS lourde demeure un facteur de très mauvais pronostic. En revanche, les facteurs directement liés à la transplantation n'ont pas été identifiés comme pronostiques $[5,6,8,9,47,50,60]$.

$\mathrm{Au}$ long cours, une prise en charge en réanimation influe sur la survie du greffon et du patient. La survie à trois ans des TRx ayant souffert d'un SDRA était à $57,8 \%$ en comparaison à $88,9 \%$ chez ceux n'ayant pas eu de SDRA [48]. Après un sepsis sévère ou un choc septique, la mortalité à cinq ans est significativement augmentée [22]. Une altération de la fonction rénale est observée chez 20 à $30 \%$ des survivants alors que 8 à $20 \%$ doivent être repris en charge en hémodialyse chronique $[5,6,9,59,61]$.

\section{Conclusion}

Moins de $10 \%$ des TRx présentent des complications sévères nécessitant une admission en réanimation. Celles-ci sont principalement de nature infectieuse entraînant des défaillances d'organes notamment respiratoire. Leur prise en charge doit tenir compte du terrain et de l'IS ; elle doit se faire en concertation avec le centre de transplantation de référence. Le pronostic de ces patients reste cependant réservé dans le court et le long terme, marqué par une mortalité en réanimation de plus de $30 \%$ et une altération de la fonction rénale ultérieure du greffon chez les survivants.

Liens d'intérêts : les auteurs déclarent ne pas avoir de lien d'intérêt.

\section{Références}

1. Wolfe RA, Ashby VB, Milford EL, Ojo AO, Ettenger RE, Agodoa LY, Held PJ, Port FK, (1999) Comparison of mortality in all patients on dialysis, patients on dialysis awaiting transplantation, and recipients of a first cadaveric transplant. N Engl J Med 341: $1725-1730$

2. Czyżewski L, Sańko-Resmer J, Wyzgał J, Kurowski A, (2014) Assessment of health-related quality of life of patients after kid- ney transplantation in comparison with hemodialysis and peritoneal dialysis. Ann Transplant 19: 576-585

3. Agence de la biomédecine - Le rapport annuel médical et scientifique 2016

4. Querard AH, Le Borgne F, Dion A, Giral M, Mourad G, Garrigue V, Rostaing L, Kamar N, Loupy A, Legendre C, Morelon E, Buron F, Foucher Y, Dantan E, (2018) Propensity score-based comparison of the graft failure risk between kidney transplant recipients of standard and expanded criteria donor grafts: toward increasing the pool of marginal donors. Am J Transplant 18: $1151-1157$

5. Klouche K, Amigues L, Massanet P, Garrigue V, Delmas S, Szwarc I, Beraud JJ, Mourad G, (2009) Outcome of renal transplant recipients admitted to an intensive care unit: a 10-year cohort study. Transplantation 87: 889-895

6. Canet E, Osman D, Lambert J, Guitton C, Heng AE, Argaud L, Klouche K, Mourad G, Legendre C, Timsit JF, Rondeau E, Hourmant M, Durrbach A, Glotz D, Souweine B, Schlemmer B, Azoulay E, (2011) Acute respiratory failure in kidney transplant recipients: a multicenter study. Crit Care Lond Engl 15: R91

7. Sadaghdar H, Chelluri L, Bowles SA, Shapiro R, (1995) Outcome of renal transplant recipients in the ICU. Chest 107: $1402-1405$

8. Mouloudi E, Massa E, Georgiadou E, Iosifidis E, Kydona C, Sgourou K, Anagnostara E, Imvrios G, Fouzas I, Papanikolaou V, Gritsi-Gerogianni N, (2012) Course and outcome of renal transplant recipients admitted to the intensive care unit: a 20 -year study. Transplant Proc 44: 2718-2720

9. Bige N, Zafrani L, Lambert J, Peraldi MN, Snanoudj R, Reuter D, Legendre C, Chevret S, Lemiale V, Schlemmer B, Azoulay E, Canet E, (2014) Severe infections requiring intensive care unit admission in kidney transplant recipients: impact on graft outcome. Transpl Infect Dis 16: 588-596

10. Canet E, Zafrani L, Azoulay É, (2016) The critically-ill kidney transplant recipient: a narrative review. Chest 149: 1546-1555

11. Berthoux F, Jones E, Gellert R, Mendel S, Saker L, Briggs D, (1999) Epidemiological data of treated end-stage renal failure in the European Union (EU) during the year 1995: report of the European Renal Association Registry and the National Registries. Nephrol Dial Transplant 14: 2332-2342

12. Sarnak MJ, Jaber BL, (2000) Mortality caused by sepsis in patients with end-stage renal disease compared with the general population. Kidney Int 58: 1758-1764

13. Lorent M, Giral M, Pascual M, Koller MT, Steiger J, TrébernLaunay K, Legendre C, Kreis H, Mourad G, Garrigue V, Rostaing L, Kamar N, Kessler M, Ladrière M, Morelon E, Buron F, Golshayan D, Foucher Y, (2016) Mortality prediction after the first year of kidney transplantation: an observational study on two European cohorts. PloS One 11: e0155278

14. Metalidis C, Kuypers D, (2011) Immunosuppressive therapy after kidney transplantation: current and new strategies. Minerva Urol Nefrol 63: 1-19

15. Hariharan S, Johnson CP, Bresnahan BA, Taranto SE, McIntosh MJ, Stablein D, (2000) Improved graft survival after renal transplantation in the United States, 1988 to 1996. N Engl J Med 342: 605-612

16. Fishman JA, (2007) Infection in solid-organ transplant recipients. N Engl J Med 357: 2601-2614

17. Pilmore H, Dent H, Chang S, McDonald SP, Chadban SJ, (2010) Reduction in cardiovascular death after kidney transplantation. Transplantation 89: 851-857

18. Jeloka TK, Ross H, Smith R, Huang M, Fenton S, Cattran D, Schiff J, Cardella C, Cole E, (2007) Renal transplant outcome in high-cardiovascular risk recipients. Clin Transplant 21: 609-614

19. Dharnidharka VR, Schnitzler MA, Chen J, Brennan DC, Axelrod D, Segev DL, Schechtman KB, Zheng J, Lentine KL, (2016) 
Differential risks for adverse outcomes 3 years after kidney transplantation based on initial immunosuppression regimen: a national study. Transpl Int 29: 1226-1236

20. Kalil AC, Syed A, Rupp ME, Chambers H, Vargas L, Maskin A, Miles CD, Langnas A, Florescu DF, (2015) Is bacteremic sepsis associated with higher mortality in transplant recipients than in nontransplant patients? A matched case-control propensityadjusted study. Clin Infect Dis 60: 216-222

21. Malinis MF, Mawhorter SD, Jain A, Shrestha NK, Avery RK, van Duin D, (2012) Staphylococcus aureus bacteremia in solid organ transplant recipients: evidence for improved survival when compared with nontransplant patients. Transplantation 93: $1045-1050$

22. Schachtner T, Stein M, Reinke P, (2018) Sepsis after renal transplantation: clinical, immunological, and microbiological risk factors. Transpl Infect Dis (doi: 10.1111/tid.12695. Epub 2017 May 9)

23. Bafi AT, Tomotani DYV, de Freitas FGR, (2017) Sepsis in solidorgan transplant patients. Shock 47: 12-16

24. Mourad G, Serre JE, Alméras C, Basel O, Garrigue V, Pernin V, Le Quintrec M, (2016) Infectious and neoplasic complications after kidney transplantation. Nephrol Ther 12: 468-487

25. Helanterä I, Schachtner T, Hinrichs C, Salmela K, Kyllönen L, Koskinen P, Lautenschlager I, Reinke P, (2014) Current characteristics and outcome of cytomegalovirus infections after kidney transplantation. Transpl Infect Dis 16: 568-577

26. Wan S, Roberts MA, Mount P, (2016) Normal saline versus lower-chloride solutions for kidney transplantation. Cochrane Database Syst Rev 8: CD010741

27. Semler MW, Self WH, Wanderer JP, Ehrenfeld JM, Wang L, Byrne DW, Stollings JL, Kumar AB, Hughes CG, Hernandez A, Guillamondegui OD, May AK, Weavind L, Casey JD, Siew ED, Shaw AD, Bernard GR, Rice TW, (2018) Balanced crystalloids versus saline in critically-ill adults. N Engl J Med 378: 829-839

28. Mårtensson J, Bellomo R, (2015) Are all fluids bad for the kidney? Curr Opin Crit Care 21: 292-301

29. Shih CJ, Tarng DC, Yang WC, Yang CY, (2014) Immunosuppressant dose reduction and long-term rejection risk in renal transplant recipients with severe bacterial pneumonia. Singapore Med J 55: 372-377

30. Tu G, Ju M, Zheng Y, Zhu D, Xu M, Rong R, Zhu T, Luo Z, (2014) An interdisciplinary approach for renal transplant recipients with severe pneumonia: a single ICU experience. Intensive Care Med 40: 914-915

31. Sun Q, Liu ZH, Chen J, Ji S, Tang Z, Cheng Z, Ji D, Li LS, (2006) An aggressive systematic strategy for acute respiratory distress syndrome caused by severe pneumonia after renal transplantation. Transpl Int 19: 110-116

32. Azoulay E, Pickkers P, Soares M, Perner A, Rello J, Bauer PR, van de Louw A, Hemelaar P, Lemiale V, Taccone FS, Martin Loeches I, Meyhoff TS, Salluh J, Schellongowski P, Rusinova K, Terzi N, Mehta S, Antonelli M, Kouatchet A, Barratt-Due A, Valkonen M, Landburg PP, Bruneel F, Bukan RB, Pène F, Metaxa V, Moreau AS, Souppart V, Burghi G, Girault C, Silva UVA, Montini L, Barbier F, Nielsen LB, Gaborit B, Mokart D, Chevret S, Efraim investigators and the Nine-I study group, (2017) Acute hypoxemic respiratory failure in immunocompromised patients: the Efraim multinational prospective cohort study. Intensive Care Med 43: 1808-1819

33. Godet C, Elsendoorn A, Roblot F, (2012) Benefit of CT scanning for assessing pulmonary disease in the immunodepressed patient. Diagn Interv Imaging 93: 425-430

34. Reichenberger F, Dickenmann M, Binet I, Solèr M, Bolliger C, Steiger J, Brunner F, Thiel G, Tamm M, (2001) Diagnostic yield of bronchoalveolar lavage following renal transplantation. Transpl Infect Dis 3: 2-7
35. Sileri P, Pursell KJ, Coady NT, Giacomoni A, Berliti S, Tzoracoleftherakis E, Testa G, Benedetti E, (2002) A standardized protocol for the treatment of severe pneumonia in kidney transplant recipients. Clin Transplant 16: 450-454

36. Amigues L, Klouche K, Massanet P, Gaillard N, Garrigue V, Beraud JJ, Mourad G, (2005) Sirolimus-associated acute respiratory distress syndrome in a renal transplant recipient. Transplant Proc 37: 2830-2831

37. Ulas A, Kaplan S, Zeyneloglu P, Torgay A, Pirat A, Haberal M, (2015) Acute respiratory failure in renal transplant recipients: a single intensive care unit experience. Exp Clin Transplant 13: $44-47$

38. Tu G, He H, Yin K, Ju M, Zheng Y, Zhu D, Luo Z, (2017) Highflow nasal cannula versus noninvasive ventilation for treatment of acute hypoxemic respiratory failure in renal transplant recipients. Transplant Proc 49: 1325-1330

39. Hilbert G, Gruson D, Vargas F, Valentino R, Gbikpi-Benissan G, Dupon M, Reiffers J, Cardinaud JP, (2001) Noninvasive ventilation in immunosuppressed patients with pulmonary infiltrates, fever, and acute respiratory failure. N Engl J Med 344: 481-487

40. Lemiale V, Mokart D, Resche-Rigon M, Pène F, Mayaux J, Faucher E, Nyunga M, Girault C, Perez P, Guitton C, Ekpe K, Kouatchet A, Théodose I, Benoit D, Canet E, Barbier F, Rabbat A, Bruneel F, Vincent F, Klouche K, Loay K, Mariotte E, Bouadma L, Moreau AS, Seguin A, Meert AP, Reignier J, Papazian L, Mehzari I, Cohen Y, Schenck M, Hamidfar R, Darmon M, Demoule A, Chevret $\mathrm{S}$, Azoulay E; Groupe de recherche en réanimation respiratoire $\mathrm{du}$ patient d'onco-hématologie (GRRR-OH), (2015) Effect of noninvasive ventilation vs oxygen therapy on mortality among immunocompromised patients with acute respiratory failure: a randomized clinical trial. JAMA 314: 1711-1719

41. Frat JP, Thille AW, Mercat A, Girault C, Ragot S, Perbet S, Prat G, Boulain T, Morawiec E, Cottereau A, Devaquet J, Nseir S, Razazi K, Mira JP, Argaud L, Chakarian JC, Ricard JD, Wittebole X, Chevalier S, Herbland A, Fartoukh M, Constantin JM, Tonnelier JM, Pierrot M, Mathonnet A, Béduneau G, DelétageMétreau C, Richard JCM, Brochard L, Robert R; FLORALI Study Group, REVA Network, (2015) High-flow oxygen through nasal cannula in acute hypoxemic respiratory failure. N Engl J Med 372: 2185-2196

42. Frat JP, Ragot S, Girault C, Perbet S, Prat G, Boulain T, Demoule A, Ricard JD, Coudroy R, Robert R, Mercat A, Brochard L, Thille AW; REVA network, (2016) Effect of non-invasive oxygenation strategies in immunocompromised patients with severe acute respiratory failure: a post hoc analysis of a randomised trial. Lancet Respir Med 4: 646-652

43. Lemiale V, Resche-Rigon M, Mokart D, Pène F, Argaud L, Mayaux J, Guitton C, Rabbat A, Girault C, Kouatchet A, Vincent F, Bruneel F, Nyunga M, Seguin A, Klouche K, Colin G, Kontar L, Perez P, Meert AP, Benoit DD, Papazian L, Demoule A, Chevret S, Azoulay E, (2017) High-flow nasal cannula oxygenation in immunocompromised patients with acute hypoxemic respiratory failure: a Groupe de recherche respiratoire en réanimation onco-hématologique study. Crit Care Med 45: e274-e280

44. Huang HB, Peng JM, Weng L, Liu GY, Du B, (2018) High-flow oxygen therapy in immunocompromised patients with acute respiratory failure: a review and meta-analysis. J Crit Care 43: 300-305

45. Azoulay E, Lemiale V, Mokart D, Nseir S, Argaud L, Pène F, Kontar L, Bruneel F, Klouche K, Barbier F, Reignier J, Berrahil-Meksen L, Louis G, Constantin JM, Mayaux J, Wallet F, Kouatchet A, Peigne V, Theodose I, Perez P, Girault C, Jaber S, Oziel J, Nyunga M, Terzi N, Bouadma L, Lebert C, Lautrette A, Bigé N, Raphalen JH, Papazian L, Darmon M, Chevret S, Demoule A, (2018) Effect of High-flow nasal oxygen vs. standard oxygen therapy on 28-Day mortality in immunocompromised patients with acute respiratory failure: The HIGH Randomized Clinical Trial. JAMA 320: 2099-2107 
46. Brunot V, Pernin V, Chartier C, Garrigue V, Vetromile F, Szwarc I, Delmas S, Portalès P, Basset D, Mourad G, (2012) An epidemic of pneumocystis jiroveci pneumonia in a renal transplantation center: role of T-cell lymphopenia. Transplant Proc 44: 2818-2820

47. Zhang P, Ye Q, Wan Q, Zhou J, (2016) Mortality predictors in recipients developing acute respiratory distress syndrome due to pneumonia after kidney transplantation. Ren Fail 38: 1082-1088

48. Shorr AF, Abbott KC, Agadoa LY, (2003) Acute respiratory distress syndrome after kidney transplantation: epidemiology, risk factors, and outcomes. Crit Care Med 31: 1325-1330

49. Cortegiani A, Madotto F, Gregoretti C, Bellani G, Laffey JG, Pham T, Van Haren F, Giarratano A, Antonelli M, Pesenti A, Grasselli G; LUNG SAFE Investigators and the ESICM Trials Group, (2018) Immunocompromised patients with acute respiratory distress syndrome: secondary analysis of the LUNG SAFE database. Crit Care Lond Engl 22: 157

50. Freitas FGR, Lombardi F, Pacheco ES, Sandes-Freitas TV de, Viana LA, Junior HTS, Medina-Pestana JO, Bafi AT, Machado FR, (2018) Clinical features of kidney transplant recipients admitted to the intensive care unit. Prog Transplant Aliso Viejo Calif 28: 56-62

51. Charbonney E, Saudan P, Triverio PA, Quinn K, Mentha G, Martin PY, (2009) Prognosis of acute kidney injury requiring renal replacement therapy in solid organ transplanted patients. Transpl Int 22: 1058-1063

52. Garg N, Rennke HG, Pavlakis M, Zandi-Nejad K, (2018) De novo thrombotic microangiopathy after kidney transplantation. Transplant Rev Orlando Fla 32: 58-68

53. Lanot E, Bouvier N, Chatelet V, Dina J, Béchade C, Ficheux M, Henri P, Lobbedez T, Hurault de Ligny B, (2016) BK virus infections in kidney transplantation. Nephrol Ther 12: 76-85

54. Wright AJ, Fishman JA, (2014) Central nervous system syndromes in solid organ transplant recipients. Clin Infect Dis 59: 1001-1011
55. Piotrowski PC, Lutkowska A, Tsibulski A, Karczewski M, Jagodziński PP, (2017) Neurologic complications in kidney transplant recipients. Folia Neuropathol 55: 86-109

56. Coussement J, Lebeaux D, van Delden C, Guillot H, Freund R, Marbus S, Melica G, Van Wijngaerden E, Douvry B, Van Laecke S, Vuotto F, Tricot L, Fernández-Ruiz M, Dantal J, Hirzel C, Jais JP, Rodriguez-Nava V, Lortholary O, Jacobs F; European Study Group for Nocardia in Solid Organ Transplantation, (2016) Nocardia infection in solid organ transplant recipients: a multicenter European case-control study. Clin Infect Dis 63: 338-345

57. Fernàndez-Sabé N, Cervera $C$, Fariñas $M C$, Bodro $M$, Muñoz $P$, Gurguí M, Torre-Cisneros J, Martín-Dávila P, Noblejas A, Len O, García-Reyne A, Del Pozo JL, Carratalà J, (2012) Risk factors, clinical features, and outcomes of toxoplasmosis in solid-organ transplant recipients: a matched case-control study. Clin Infect Dis 54: $355-361$

58. Paya CV, Fung JJ, Nalesnik MA, Kieff E, Green M, Gores G, Habermann TM, Wiesner PH, Swinnen JL, Woodle ES, Bromberg JS, (1999) Epstein-Barr virus-induced posttransplant lymphoproliferative disorders. ASTS/ASTP EBV-PTLD Task Force and The Mayo Clinic Organized International Consensus Development Meeting. Transplantation 68: 1517-1525

59. Donnelly JP, Locke JE, MacLennan PA, McGwin G, Mannon RB, Safford MM, Baddley JW, Muntner P, Wang HE, (2016) Inpatient mortality among solid organ transplant recipients hospitalized for sepsis and severe sepsis. Clin Infect Dis 63: 186-194

60. Arulkumaran N, West S, Chan K, Templeton M, Taube D, Brett SJ, (2012) Long-term renal function and survival of renal transplant recipients admitted to the intensive care unit. Clin Transplant 26: E24-E31

61. Badin J, Longuet H, Guillon A, Barbet C, Halimi JM, Lebranchu Y, Perrotin D, Buchler M, (2012) Renal function of renal transplantation patients after hospitalization in an intensive care unit. Transplant Proc 44: 2792-2794 